\title{
Graphene oxide hybrid with sulphur-nitrogen polymer for high-performance pseudocapacitors
}

\author{
Samanta Witomska, ${ }^{\dagger} \ddagger \S$ Zhaoyang Liu, ${ }^{\dagger}$ Włodzimierz Czepa, ${ }^{\ddagger}$ Alessandro Aliprandi, ${ }^{\dagger}$ Dawid Pakul- \\ ski, ${ }^{\dagger \ddagger}$ Piotr Pawluć, $₫ \S$ Artur Ciesielski* ${ }^{\dagger \ddagger}$ and Paolo Samorì*† \\ † Université de Strasbourg, CNRS, ISIS, 8 alleé Gaspard Monge, 67000 Strasbourg, France \\ $¥$ Center for Advanced Technologies, Adam Mickiewicz University, Umultowska 89c, 61614 Poznań, Poland \\ $\S$ Faculty of Chemistry, Adam Mickiewicz University, Umultowska 89b, 61614 Poznań, Poland
}

\begin{abstract}
Towards the introduction of fast faradaic pseudocapacitive behavior and the increase of the specific capacitance of carbon-based electrodes, we have covalently functionalized graphene oxide with a redox active thiourea-formaldehyde polymer yielding a multifunctional hybrid system. The multiscale physical and chemical characterization of the novel 3-dimensional hybrid revealed high material's porosity with high specific surface area $\left(402 \mathrm{~m}^{2} \mathrm{~g}^{-1}\right)$ and homogeneous element distribution. The presence of multiple functional groups comprising sulphur, nitrogen and oxygen, provide additional contribution of Faradic redox reaction in supercapacity performance, leading to a high effective electrochemical pseudocapacitance. Significantly, our graphene-based 3-dimensional thiourea-formaldehyde hybrid (G3DTF) exhibited specific capacitance as high as $400 \mathrm{~F} \mathrm{~g} \mathrm{~g}^{-1}$, areal capacitance of $160 \mathrm{mF}$ $\mathrm{cm}^{-2}$ and an energy density of $11.1 \mathrm{mWh} \mathrm{cm}^{-3}$ at scan rate of $1 \mathrm{mV} \mathrm{s}^{-1}$ with great capacitance retention (100\%) after $5000 \mathrm{cycles}$ at scan rate of $100 \mathrm{mV} \mathrm{s}^{-1}$.
\end{abstract}

\section{INTRODUCTION}

The nowadays ever-increasing energy consumption urgently calls for a sustainable energy-management through the optimized generation and storage of energy. Next generation of energy storage technologies should match stringent requirements in terms of limited size and weight to enable the operation of miniaturized electronics for mobile applications such as wearable devices and portable sensors. ${ }^{1-3}$ Among various energy storage devices, supercapacitors have recently attracted significant attention since they combine high power density, ultrafast charge-discharge rate, long cycle life, great stability and lowmaintenance cost..$^{4-7}$ According to the charge storage mechanism, supercapacitors can be classified as electrochemical double-layer capacitors, pseudocapacitors or hybrid capacitors. ${ }^{8-9}$ Many factors should be considered and optimized when designing a device for capacitance applications including the material's morphology, pore structure, electrical conductivity, specific surface area and heteroatom doping. ${ }^{10-11}$ The introduction of heteroatoms (e.g. N, O, S, P) can significantly increase the capacity performance of carbon materials by contributing with the additional pseudocapacitance through surface Faradic redox reactions. ${ }^{10}$ Therefore, the cyclic voltammetry $(\mathrm{CV})$ of such hybrid materials can be characterized as quasi-rectangular with broad redox waves proving coexistence of electric double layer capacitance and additional redox pseudocapacitance. ${ }^{12-14}$ The controlled polymer functionalization of graphene is used for the development of efficient energy conversion and storage devices. ${ }^{15-17}$ Graphene-based materials chemically doped with heteroatoms have been recently widely explored as active systems for energy storage performance as they exhibit high gravimetric capacitance ranging up $500 \mathrm{~F} \mathrm{~g}^{-1} \cdot{ }^{18-24}$ Currently, some bottlenecks in graphene based supercapacitor materials for supercapacitor need to be addressed such as: $i$ ) the lack of fast Faradic pseudocapacitive behavior, which results in low specific capacitance, and $i$ ) the restacking of graphene sheets through the strong $\pi-\pi$ interactions, which can severely decrease the accessible electrochemical surfaces, thus hindering the ion diffusion ultimately limiting the overall electrochemical performance. Graphene oxide (GO) combines the unique characteristics of graphene and related 2D materials such as the high mechanical strength $^{25}$ and large surface area ${ }^{26-27}$ with an easy, low-cost and scalable production. ${ }^{28}$ Furthermore the presence of oxygen-rich functionalities endows excellent water dispersibility and it opens various options for its covalent/non-covalent functionalization. ${ }^{29}$ The latter can be employed for the fine-tuning of GO's electrochemical properties. ${ }^{30-32}$ In fact, while it is notorious that pristine GO is an electrical insulator, in its reduced form, i.e. reduced graphene oxide ( $\mathrm{rGO}$ ), it displays conductivities reaching sheet resistances of a few tens of $\Omega \mathrm{sq}^{-1} \cdot{ }^{33}$ Noteworthy, despite the most common approach for obtaining rGO relies on the chemical reduction of $\mathrm{GO}$, a few recent reports revealed that the direct functionalization of $\mathrm{GO}$ with reducing molecules might also provide a decrease of oxygen content along with an improvement of the material's conductivity. ${ }^{34-37}$

Thiourea-based small molecules have been recently used for simultaneous functionalization and reduction of graphene oxide (GO) leading to sulphur-rich hybrids. ${ }^{36}$ In such materials the high supercapacitive performance is obtained via redox mechanism of thiocarboxylic acid ester to sulphone with the aid of external current. ${ }^{36}$ In order to leverage the performance of the 
sulphur-rich GO-based hybrids ${ }^{36}$ a plausible solution relies on increasing the amount of the thiourea groups on the GO surface by using a thiourea based polymer.

Graphene with its excellent electrical conductivity, high surface-to-volume ratio, and outstanding intrinsic double-layer capacitance is extremely attractive for high-power supercapacitors. However, the performances of current graphene-based supercapacitors are still limited, mostly due to the lack of the fast Faradaic pseudocapacitive behaviour. To tackle this issue, we have exploited a bottom-up approach towards the generation of $3 \mathrm{D}$ porous hybrid material (G3DTF) based on thiourea-formaldehyde polymer (TF) with well-defined molecular weight covalently grafted to GO (see Scheme 1). The combination of pseudocapacitive TF and GO resulted in a novel type of electrode material for supercapacitors, which revealed performance reaching the state-of-the-art of GO-based materials.

\section{RESULTS AND DISCUSSION:}

Towards the synthesis of thiourea-formaldehyde GO-based hybrid (G3DTF), we firstly prepared the thiourea-formaldehyde polymer by hydroxyl-methylation and condensation reaction between thiourea and formaldehyde (see experimental procedures in Supporting Information). ${ }^{38}$ The polymer has been characterized by means of ${ }^{1} \mathrm{HNMR}$ spectroscopy, Fourier-transform infrared spectroscopy (FT-IR) and elemental analysis (see SI). The weight-average molecular weights $\left(\mathrm{M}_{\mathrm{w}}\right)$ of thiourea-formaldehyde polymer (TF), as determined by diffusion-ordered NMR spectroscopy (DOSY NMR), amounted to $969 \mathrm{~g} \mathrm{~mol}^{-1}$ (for details see SI).

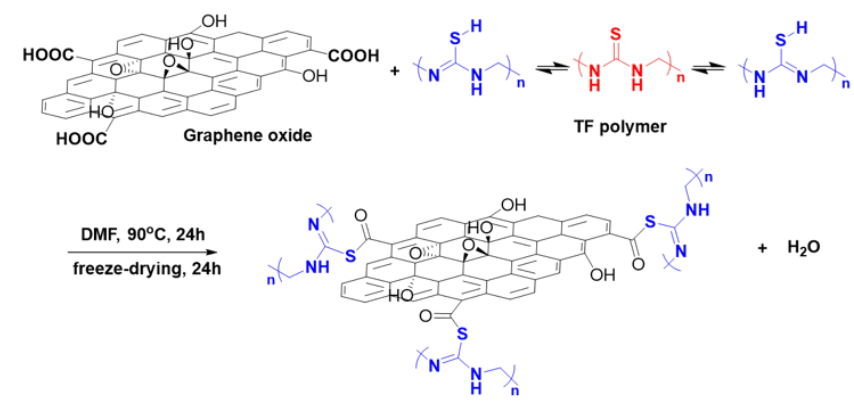

Scheme 1. Synthesis of G3DTF. Schematic representation of thiolcarboxylic acid esterification reactions of graphene oxide $(\mathrm{GO})$ and thiourea-formaldehyde polymer (TF).

Noteworthy, carboxylic groups exposed at the edges of GO sheets can be transformed to thiocarboxylic acid ester group. In particular, the thiourea group, which can exist as resonance structure, either in thione $(\mathrm{C}=\mathrm{S})$ or in thiol $(\mathrm{C}-\mathrm{S}-\mathrm{H})$, can react with the carboxylic acids on the GO sheet through thiol-carboxylic acid esterification. ${ }^{36,39}$ The functionalization of GO with TF polymer was carried out by heating commercially available aqueous dispersion of GO in the presence of TF in DMF overnight (for details see SI). Unreacted TF molecules were washed away and the resulting G3DTF was then freeze-dried for $24 \mathrm{~h}$ to sustain the high porosity and specific surface area. G3DTF was collected as a black solid. A scheme of the synthesis method employed for the chemical tailoring of GO is displayed in Scheme 1.

X-ray photoelectron spectroscopy (XPS) offered insight into the chemical composition of materials by identifying the relevant chemical elements present in both GO and G3DTF. The significant differences between the carbon, oxygen, nitrogen and sulphur peaks provided evidence for the generation of a covalent bond between the oxygen-containing functional groups on the surface of GO and thiol groups from the TF polymer (see XPS analysis in Fig. 1 and Fig. S5-6, SI). Figure S5 reveals that in the wide energy spectrum of the pristine GO there are only two peaks at $288.9 \mathrm{eV}$ and $535.2 \mathrm{eV}$ which are attributed to $\mathrm{C} 1 \mathrm{~s}$ and $\mathrm{O} 1 \mathrm{~s}$, respectively. ${ }^{40}$
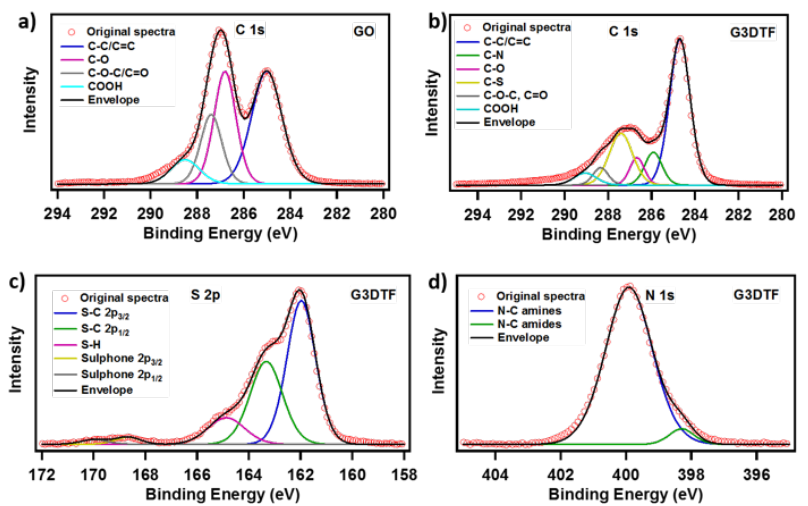

Figure 1. X-ray Photoelectron Spectroscopy characterization. High-resolution C1s spectra of (a) GO, and (b) G3DTF. (c) S2p spectra of G3DTF, and (d) N1s spectra of G3DTF.

In contrast, G3DTF spectrum displays the typical peaks of $\mathrm{N} 1 \mathrm{~s}, \mathrm{~S} 2 \mathrm{~s}$ and S2p at $399.9 \mathrm{eV}, 226.7 \mathrm{eV}$ and $162.4 \mathrm{eV}$, respectively. After functionalization, the nitrogen and sulphur content increased up to $15,1 \%$ and $5.2 \%$, while the $\mathrm{O}$ content dropped from $33.5 \%$ for GO to $11.6 \%$ for G3DTF as a result of the thiolcarboxylic acid esterification reaction and partial reduction of GO. ${ }^{41-42}$ In particular, the C1s XPS spectrum of the G3DTF shows a decrease in the intensity of the peaks $\mathrm{C}-\mathrm{O}(286.4 \mathrm{eV})$, $\mathrm{C}-\mathrm{O}-\mathrm{C} / \mathrm{C}=\mathrm{O}(288.06 \mathrm{eV})$ and $\mathrm{O}=\mathrm{C}-\mathrm{OH}(288.8 \mathrm{eV})$ upon functionalization, and the emergence of new components in the $\mathrm{C} 1 \mathrm{~s}$ spectrum of G3DTF at $285.6 \mathrm{eV}$ and $287.3 \mathrm{eV}$ which are attributed to C-N and C-S bonds, respectively. Moreover, the G3DTF's $S 2 p$ core level line exhibits $S 2 p_{3 / 2}$ and $S 2 p_{1 / 2}$ signals at $161.9 \mathrm{eV}$ and $163.3 \mathrm{eV}$, respectively, as well as a trace peaks at $168.7 \mathrm{eV}$ and $169.9 \mathrm{eV}$. The intensity ratio of the $\mathrm{S} 2 \mathrm{p}_{3 / 2}$ and $\mathrm{S} 2 \mathrm{p}_{1 / 2}$ peaks is $2: 1$ and $1.2 \mathrm{eV}$ binding energy separation correspond to spin-orbital splitting of sulphur atoms of TF. The trace peak at $168.5 \mathrm{eV}$ is located at the position predicted for sulfone groups. ${ }^{36}$ Additionally, in the S2p spectrum a signal indicating the presence of S-H bond at $164.8 \mathrm{eV}$ is observed, which correspond to the resonance structures of thiourea derivatives (see Fig. 1).

Fourier transform infrared (FTIR) spectroscopy was employed to bestow more information onto the structure of G3DTF. The spectrum of GO (Fig. S4) displays typical bands associated to $\mathrm{C}=\mathrm{O}$ stretching at $1724 \mathrm{~cm}^{-1}, \mathrm{C}=\mathrm{C}$ stretching at $1624 \mathrm{~cm}^{-1}, \mathrm{C}-\mathrm{O}$ stretching of epoxy groups at $1216 \mathrm{~cm}^{-1}$ and $1054 \mathrm{~cm}^{-1}$ and a broad peak between 3000 and $3500 \mathrm{~cm}^{-1}$ corresponding to $\mathrm{O}-\mathrm{H}$ vibration. ${ }^{43}$ The FTIR spectrum of G3DTF 
exhibits several bands characteristic for TF functional groups introduced upon the GO's functionalization. A strong absorption peak at $1533 \mathrm{~cm}^{-1}$ (not observed in the neat TF) is assigned to $\mathrm{C}=\mathrm{N}-\mathrm{H}$ stretching vibration, which indicates that upon covalent attachment of TF to GO, the resonant structure of the former is shifted towards thiol containing form. Such observation was confirmed by S2p XPS spectra of TF and G3DTF. The S2p spectrum of TF was measured as a control (Fig. S7). The formation of the G3DTF composite was further evidenced by the appearance of spectral features indicating the presence of $\mathrm{N}-\mathrm{H}$ $\left(3315 \mathrm{~cm}^{-1}\right.$ and $\left.3034 \mathrm{~cm}^{-1}\right)$, N-C $\left(1439 \mathrm{~cm}^{-1}\right.$ and $\left.1319 \mathrm{~cm}^{-1}\right), \mathrm{C}-$ $\mathrm{S}\left(959 \mathrm{~cm}^{-1}\right.$ and $\left.650 \mathrm{~cm}^{-1}\right)$, which were not present in the spectrum of neat GO. Raman spectroscopy provides valuable information regarding the quality and degree of functionalization of graphene and its derivatives by taking advantage of its highest sensitivity to the electronic structure of the carbon nanostructures, therefore the degree of hybridization, the crystal disorder and the extent of chemical modification. ${ }^{44}$ Figure $2 \mathrm{~b}$ displays the Raman spectra of GO and G3DTF. The efficient functionalization was confirmed by following the ratio between $\mathrm{D}$ and $\mathrm{G}$ peaks of GO and G3DTF ${ }^{45}$ which are located at ca. 1350 $\mathrm{cm}^{-1}$ and $1600 \mathrm{~cm}^{-1}$, respectively. ${ }^{46}$ In particular, the increase in the $\mathrm{I}_{\mathrm{D}} / \mathrm{I}_{\mathrm{G}}$ ratio from 1.00 to 1.21 in G3DTF reflects the enhancement in structural and electronic disorder after grafting $\mathrm{TF}$, which is due to the increase of the $\mathrm{sp}^{3}$ carbon atoms in the hybrid.

The morphology of G3DTF was analysed by means of scanning electron microscope (SEM). Figure 2 a shows the homogenous porous structure of G3DTF composite. Furthermore, energy-dispersive X-ray spectroscopy (EDX) analysis (Figure 2c) shows the uniform distribution of TF component within the G3DTF.

The average interlayer distance in GO and G3DTF powder were studied by wide-angle X-ray scattering (WAXS). XRD pattern of the pristine GO displays only a typical sharp peak at $\approx 10.01^{\circ}$ (see Fig. S3), corresponding to an interlayer spacing of $0.87 \mathrm{~nm}$ due to the $(002)$ reflection of stacked GO sheets, in accordance with the previously reported values. ${ }^{35,47}$ Conversely, the XRD spectra of G3DTF exhibited peaks at wide range of angles $\left(17.5^{\circ}-36.5^{\circ}\right)$ highlighting great similarity to XRD pattern of pristine TF polymer and indicating substantial changes in the interlayer distance if compared that of GO (Fig S3).

The porosity of G3DTF was quantified by $\mathrm{N}_{2}$ adsorption-desorption isotherms. The accessible surface area of the starting material of GO calculated with Brunauer-Emmett-Teller (BET) model amounted to $106 \mathrm{~m}^{2} \mathrm{~g}^{-1}$. Conversely, upon functionalization with TF, it significantly increased up to $403 \mathrm{~m}^{2} \mathrm{~g}^{-1}$. The average pore diameter calculated with the Barrett-JoynerHalenda (BJH) model resulted being close to $14.7 \mathrm{~nm}$ (see Tab. S2, Fig. S8-S9). $\mathrm{N}_{2}$ adsorption and desorption isotherms of G3DTF are similar to type IV isotherm, which exhibits a distinct $\mathrm{H} 3$ hysteresis loop, indicating a mesoporous nature of G3DTF. This type of isotherm is often used to describe adsorption-desorption process for $2 \mathrm{D}$ and $3 \mathrm{D}$-carbon based materials $^{48-49}$

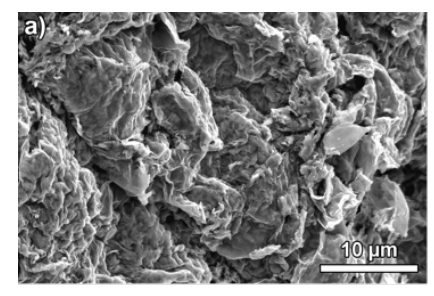

b)

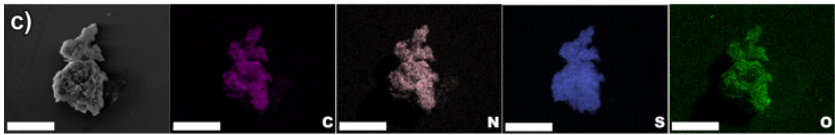

Figure 2. Morphological, compositional and electronic characterization. (a) SEM image of G3DTF; (b) Raman spectra of GO and G3DTF; (c) SEM image of G3DTF and EDX elemental mapping of carbon $(\mathrm{C})$, oxygen $(\mathrm{O})$, nitrogen $(\mathrm{N})$ and sulphur $(\mathrm{S})$, scale bars correspond to $20 \mu \mathrm{m}$.

The incorporation of thiourea polymer into graphene sheets can effectively improve the electrochemical properties of the 2D material. Notably it can reduce self-agglomeration and consequently increase the specific surface area and further enhance the specific capacitance of graphene materials. Moreover, the introduction of heteroatoms enriches the electronic property of the material resulting in the appearance of pseudocapacitance in the system by redox reactions involving sulphone groups. ${ }^{50-51}$ The potential use of this composite as electrode materials for supercapacitors was tested by standard cyclic voltammetry (CV). All electrochemical measurements were conducted in a three-electrode cell, and the working electrode was prepared as free-standing film sandwiched between two Pt meshes based on G3DTF and electrochemically exfoliated graphene (EEG) ${ }^{24,52}$ as a conducting additive. The Figure S1 in Supporting Information shows G3DTF:EG film with lateral size of $1 \times 1 \mathrm{~cm}^{2}$ and film thickness of $20 \mu \mathrm{m}$. G3DTF is expected to be promising electrode material for supercapacitors, due to the presence of both pseudocapacitive thiourea-formaldehyde polymer and electron-double-layer-capacitive graphene forming a hybrid porous structure. The pseudocapacitive mechanism of sulphur functionalized graphene has been proposed by Lee et al. ${ }^{36}$, on the basis of redox reaction between thiocarboxylic acid ester group and sulphone. Thiocarboxylic acid ester functional groups chemically grafted onto the graphene materials can be electrochemically oxidized to sulphone, which can be followed by electrochemical reduction back to thiocarboxylic acid ester (Scheme 2).

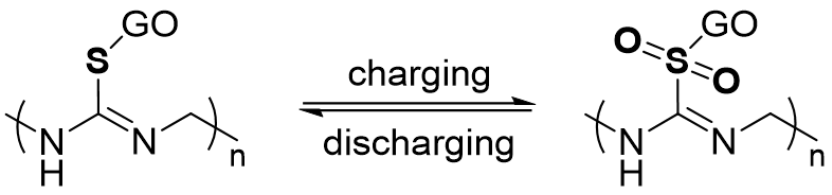

Scheme 2. The pseudocapacitative mechanism of redox reaction between thiocarboxylic acid ester and sulphone group on the surface of G3DTF.

Figure 3 a-e shows the cyclic voltammetry (CV) curves of the supercapacitor electrode based on G3DTF nanosheets. The CV profiles exhibit certain electrical double layer behaviour between the voltage potential of 0 and $1 \mathrm{~V}$ at scan rates 1 to $10^{4}$ $\mathrm{mV} \mathrm{s}^{-1}$, indicating the capacitance contribution from graphene. Meanwhile, the CV curves exhibit typical pseudocapacitive behaviour of thiourea-formaldehyde with strong redox peaks in 
the range of 0 to $1 \mathrm{~V}$ at scan rates below $100 \mathrm{mV} \mathrm{s}^{-1}$. Figure $3 \mathrm{f}$ plots the calculated specific capacitance of G3DTF nanosheets versus scan rates. Remarkably, the G3DTF nanosheets delivered an impressive specific capacitance of $400 \mathrm{~F} \mathrm{~g} \mathrm{~g}^{-1}$, areal capacitance of $160 \mathrm{mF} \mathrm{cm}^{-2}$ and an energy density of $11.1 \mathrm{mWh}$ $\mathrm{cm}^{-3}$ at $1 \mathrm{mV} \mathrm{s}^{-1}$, and outperforming most state-of-the-art graphene-based polymer electrode materials (see Table S5).
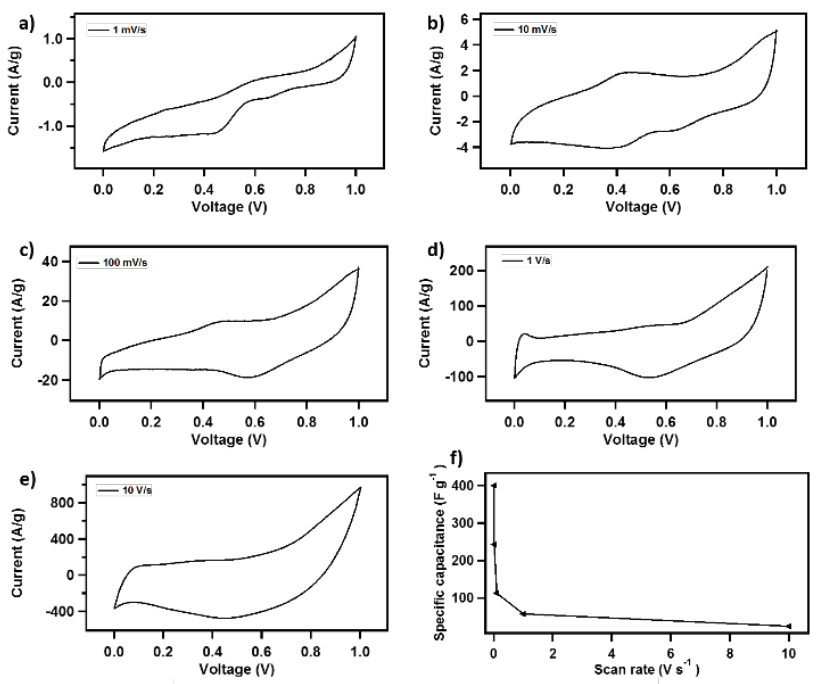

g)

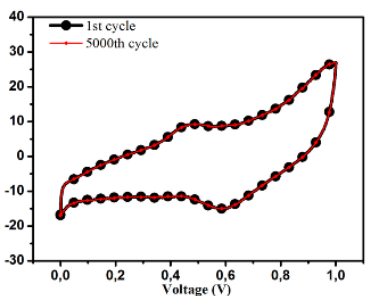

Figure 3. a-e) CV curves of G3DTF as supercapacitor electrode material at scan rates ranging from 1 to $10^{4} \mathrm{mV} \mathrm{s}^{-1}, \mathrm{f}$ ) the evolution of G3DTF specific capacitances versus scan rates and g) Nearly overlapped CV curves of G3DTF supercapacitor electrode at $1^{\text {st }}$ and $5000^{\text {th }}$ cycle (scan rate $100 \mathrm{mV} \mathrm{s}^{-1}$ ), demonstrating excellent stability.

Especially, the specific capacitance remained $113 \mathrm{~F} \mathrm{~g}^{-1}$ at a high scan rate of $100 \mathrm{mV} \mathrm{s}^{-1}$, showing a capacitance retention of $28 \%$. Such excellent rate capability might be attributed to the unique porous structure of G3DTF as monitored in Figure 3a, demonstrating the fast ion diffusion and high-charge storage behaviour. The capacitance drop at higher scan rates can be attributed to the inaccessible porous structure for the electrolyte. Moreover, G3DTF electrode exhibit superior cycling stability with capacitance retention reaching $100 \%$ after 5000 cycles at a scan rate of $100 \mathrm{mV} \mathrm{s}^{-1}$ (see Fig. $3 \mathrm{~g}$,). Such excellent stability after long-term cycling suggests that the porous structure of G3DTF can well tolerate large volumetric change and counter ion drain effect during the rapid charging/discharging process, which makes G3DTF highly promising for future supercapacitor applications.

\section{CONCLUSIONS:}

In conclusion, we have demonstrated a facile approach to the bottom-up fabrication of a novel 3D sulphur-nitrogen functionalized graphene oxide material, obtained by covalently grafting thiourea-formaldehyde polymer onto GO by thiol-carboxylic acid esterification reaction. The obtained material was characterized in terms of morphology, chemical composition, pore structure and surface area and tested toward its electrochemical potential in pseudosupercapacity measurements. Modification of GO with TF lead to increased capacitance and energy density of the electrodes for supercapacitor applications due to the synergistic effect of graphene and sulphur-rich polymer. High specific capacitances and good cycling stability were achieved for G3DTF with the highest specific capacitance of $400 \mathrm{~F} \mathrm{~g}^{-1}$, areal capacitance of $160 \mathrm{mF} \mathrm{cm}^{-2}$ and an energy density of $11.1 \mathrm{mWh}$ $\mathrm{cm}^{-3}$ at the lowest scan rate, $1 \mathrm{mV} \mathrm{s}^{-1}$ with great stability and durability. The concept of combining both pseudocapacitive thiourea-based polymer and electron-double-layer-capacitive graphene offers promising opportunities for future portable and wearable power supplies in diverse applications.

\section{ASSOCIATED CONTENT}

Supporting Information. Experimental section, figures of XRD, FTIR, XPS, BET surface area, cyclic voltammetry, elemental analysis, DOSY NMR, and state-of-the-art graphene-based polymer electrode materials.

"This material is available free of charge via the Internet at http://pubs.acs.org."

\section{AUTHOR INFORMATION}

\section{Corresponding Author}

*samori@unistra.fr

* ciesielski@unistra.fr

Notes

The authors declare no competing financial interests.

\section{ACKNOWLEDGMENT}

This work was supported by the European Commission through the Graphene Flagship Core 2 project (GA No. 785219), the Polish National Science Centre (Grant no. 2016/23/N/ST5/00063 and Grant no. 2015/18/E/ST5/00188), the ANR Labex project CSC (ANR10- LABX-0026 CSC) within the Investissement d'Avenir program ANR-10-IDEX-0002-02, the International Center for Frontier Research in Chemistry (icFRC) and grant no. POWR.03.02.0000-I023/17 co-financed by the European Union through the European Social Fund under the Operational Program Knowledge Education Development.

\section{REFERENCES}

1. Lee, H.; Choi, T. K.; Lee, Y. B.; Cho, H. R.; Ghaffari, R.; Wang, L.; Choi, H. J.; Chung, T. D.; Lu, N.; Hyeon, T.; Choi, S. H.; Kim, D.-H., A graphene-based electrochemical device with thermoresponsive microneedles for diabetes monitoring and therapy. Nat. Nanotechnol. 2016, 11, 566.

2. Kou, L.; Huang, T.; Zheng, B.; Han, Y.; Zhao, X.; Gopalsamy, K.; Sun, H.; Gao, C., Coaxial wet-spun yarn supercapacitors for high-energy density and safe wearable electronics. Nat. Commun. 2014, 5, 3754.

$3 . \quad$ Anichini, C.; Czepa, W.; Pakulski, D.; Aliprandi, A.; Ciesielski, A.; Samorì, P., Chemical sensing with 2D materials. Chem. Soc. Rev. 2018, 47 (13), 4860-4908.

4. $\quad$ Wang, Y.; Shi, Z. Q.; Huang, Y.; Ma, Y. F.; Wang, C. Y.; Chen, M. M.; Chen, Y. S., Supercapacitor Devices Based on 
Graphene Materials. J. Phys. Chem. C 2009, 113 (30), 1310313107.

5. Huang, Y.; Liang, J.; Chen, Y., An overview of the applications of graphene-based materials in supercapacitors. Small 2012, 8 (12), 1805-34.

6. $\quad$ Simon, P.; Gogotsi, Y.; Dunn, B., Materials science. Where do batteries end and supercapacitors begin? Science 2014, 343 (6176), 1210-1.

7. Yu, Z. N.; Tetard, L.; Zhai, L.; Thomas, J., Supercapacitor electrode materials: nanostructures from 0 to 3 dimensions. Energy Environ. Sci. 2015, 8 (3), 702-730.

8. Simon, P.; Gogotsi, Y., Materials for electrochemical capacitors. Nat. Mater. 2008, 7 (11), 845-54.

9. Bonaccorso, F.; Colombo, L.; Yu, G.; Stoller, M.; Tozzini, V.; Ferrari, A. C.; Ruoff, R. S.; Pellegrini, V., 2D materials. Graphene, related two-dimensional crystals, and hybrid systems for energy conversion and storage. Science 2015, 347 (6217), 1246501.

10. Wang, Q.; Yan, J.; Fan, Z. J., Carbon materials for high volumetric performance supercapacitors: design, progress, challenges and opportunities. Energy Environ. Sci. 2016, 9 (3) 729-762.

11. Mendoza-Sanchez, B.; Gogotsi, Y., Synthesis of TwoDimensional Materials for Capacitive Energy Storage. Adv. Mater. 2016, 28 (29), 6104-35.

12. Tian, W. Q.; Gao, Q. M.; Tan, Y. L.; Zhang, Y. L.; Xu, J. D.; Li, Z. Y.; Yang, K.; Zhu, L. H.; Liu, Z. P., Three-dimensional functionalized graphenes with systematical control over the interconnected pores and surface functional groups for high energy performance supercapacitors. Carbon 2015, 85, 351-362.

13. Zhu, Y.; Murali, S.; Stoller, M. D.; Ganesh, K. J.; Cai, W.; Ferreira, P. J.; Pirkle, A.; Wallace, R. M.; Cychosz, K. A.; Thommes, M.; Su, D.; Stach, E. A.; Ruoff, R. S., Carbon-Based Supercapacitors Produced by Activation of Graphene. Science 2011, 332 (6037), 1537-1541.

14. Kannappan, S.; Yang, H.; Kaliyappan, K.; Manian, R. K.; Pandian, A. S.; Lee, Y. S.; Jang, J. H.; Lu, W., Thiolated-graphenebased supercapacitors with high energy density and stable cycling performance. Carbon 2018, 134, 326-333.

15. Dai, L., Functionalization of Graphene for Efficient Energy Conversion and Storage. Acc. Chem. Res. 2013, 46 (1), 3142.

16. Huang, X.; Qi, X.; Boey, F.; Zhang, H., Graphene-based composites. Chem. Soc. Rev. 2012, 41 (2), 666-686.

17. Layek, R. K.; Nandi, A. K., A review on synthesis and properties of polymer functionalized graphene. Polymer 2013, 54 (19), 5087-5103

18. Yu, X.; Kang, Y.; Park, H. S., Sulfur and phosphorus codoping of hierarchically porous graphene aerogels for enhancing supercapacitor performance. Carbon 2016, 101, 49-56.

19. Wang, X.; Zhang, Y.; Zhi, C.; Wang, X.; Tang, D.; Xu, Y.; Weng, Q.; Jiang, X.; Mitome, M.; Golberg, D.; Bando, Y., Three-dimensional strutted graphene grown by substrate-free sugar blowing for high-power-density supercapacitors. Nat. Commun. 2013, 4, 2905.

20. Hu, J.; Kang, Z.; Li, F.; Huang, X., Graphene with threedimensional architecture for high performance supercapacitor. Carbon 2014, 67, 221-229.

21. Seredych, M.; Bandosz, T. J., S-doped micro/mesoporous carbon-graphene composites as efficient supercapacitors in alkaline media. J. Mater. Chem. A 2013, 1 (38), 11717.

22. Wu, Z. S.; Parvez, K.; Winter, A.; Vieker, H.; Liu, X.; Han, S.; Turchanin, A.; Feng, X.; Müllen, K., Layer-by-layer assembled heteroatom-doped graphene films with ultrahigh volumetric capacitance and rate capability for microsupercapacitors. Adv. Mater. 2014, 26 (26), 4552-8.
23. Wang, N.; Han, G.; Song, H.; Xiao, Y.; Li, Y.; Zhang, Y.; Wang, H., Integrated flexible supercapacitor based on poly (3, 4-ethylene dioxythiophene) deposited on $\mathrm{Au} /$ porous polypropylene film/Au. J. Power Sources 2018, 395, 228-236.

24. Liu, Z.; Liu, S.; Dong, R.; Yang, S.; Lu, H.; Narita, A.; Feng, X.; Müllen, K., High Power In-Plane Micro-Supercapacitors Based on Mesoporous Polyaniline Patterned Graphene. Small 2017, 13 (14), 1603388.

25. Dikin, D. A.; Stankovich, S.; Zimney, E. J.; Piner, R. D.; Dommett, G. H. B.; Evmenenko, G.; Nguyen, S. T.; Ruoff, R. S., Preparation and characterization of graphene oxide paper. Nature 2007, 448, 457.

26. Lv, W.; Tang, D. M.; He, Y. B.; You, C. H.; Shi, Z. Q.; Chen, X. C.; Chen, C. M.; Hou, P. X.; Liu, C.; Yang, Q. H., Lowtemperature exfoliated graphenes: vacuum-promoted exfoliation and electrochemical energy storage. ACS Nano 2009, 3 (11), 37306.

27. Zhu, Y.; Murali, S.; Stoller, M. D.; Velamakanni, A.; Piner, R. D.; Ruoff, R. S., Microwave assisted exfoliation and reduction of graphite oxide for ultracapacitors. Carbon 2010, 48 (7), 2118-2122.

28. Tung, V. C.; Allen, M. J.; Yang, Y.; Kaner, R. B., Highthroughput solution processing of large-scale graphene. Nat. Nanotechnol. 2008, 4, 25.

29. Georgakilas, V.; Otyepka, M.; Bourlinos, A. B.; Chandra, V.; Kim, N.; Kemp, K. C.; Hobza, P.; Zboril, R.; Kim, K. S., Functionalization of Graphene: Covalent and Non-Covalent Approaches, Derivatives and Applications. Chem. Rev. 2012, 112 (11), 6156-6214.

30. Li, D.; Müller, M. B.; Gilje, S.; Kaner, R. B.; Wallace, G. G., Processable aqueous dispersions of graphene nanosheets. Nat. Nanotechnol. 2008, 3 (2), 101-5.

31. Kim, J.; Cote, L. J.; Kim, F.; Yuan, W.; Shull, K. R.; Huang, J., Graphene oxide sheets at interfaces. J. Am. Chem. Soc. 2010, 132 (23), 8180-6.

32. Kim, F.; Cote, L. J.; Huang, J., Graphene oxide: surface activity and two-dimensional assembly. Adv. Mater. 2010, 22 (17), 1954-8.

33. Dreyer, D. R.; Park, S.; Bielawski, C. W.; Ruoff, R. S., The chemistry of graphene oxide. Chem. Soc. Rev. 2010, 39 (1), 228-40.

34. Stankovich, S.; Dikin, D. A.; Piner, R. D.; Kohlhaas, K. A.; Kleinhammes, A.; Jia, Y.; Wu, Y.; Nguyen, S. T.; Ruoff, R. S., Synthesis of graphene-based nanosheets via chemical reduction of exfoliated graphite oxide. Carbon 2007, 45 (7), 1558-1565.

35. Zhang, K.; Zhang, L. L.; Zhao, X. S.; Wu, J. S., Graphene/Polyaniline Nanofiber Composites as Supercapacitor Electrodes. Chem. Mater. 2010, 22 (4), 1392-1401.

36. Lee, W. S. V.; Leng, M.; Li, M.; Huang, X. L.; Xue, J. M., Sulphur-functionalized graphene towards high performance supercapacitor. Nano Energy 2015, 12 (Supplement C), 250-257.

37. Song, S.; Xue, Y.; Feng, L.; Elbatal, H.; Wang, P.; Moorefield, C. N.; Newkome, G. R.; Dai, L., Reversible selfassembly of terpyridine-functionalized graphene oxide for energy conversion. Angew. Chem. Int. Ed. Engl. 2014, 53 (5), 1415-9.

38. Liu, S. J.; Guo, Y. P.; Yang, H. Y.; Wang, S.; Ding, H.; Qi, Y., Synthesis of a water-soluble thiourea-formaldehyde (WTF) resin and its application to immobilize the heavy metal in MSWI fly ash. J. Environ. Manage. 2016, 182 (Supplement C), 328-334. 39. Yang, J.; Gong, D.; Li, G.; Zeng, G.; Wang, Q.; Zhang, Y.; Liu, G.; Wu, P.; Vovk, E.; Peng, Z.; Zhou, X.; Yang, Y.; Liu, Z.; Sun, Y., Self-Assembly of Thiourea-Crosslinked Graphene Oxide Framework Membranes toward Separation of Small Molecules. Adv. Mater. 2018, 30 (16), e1705775.

40. Yang, D.; Velamakanni, A.; Bozoklu, G.; Park, S.; Stoller, M.; Piner, R. D.; Stankovich, S.; Jung, I.; Field, D. A.; Ventrice, C. A.; Ruoff, R. S., Chemical analysis of graphene oxide 
films after heat and chemical treatments by X-ray photoelectron and Micro-Raman spectroscopy. Carbon 2009, 47 (1), 145-152.

41. Satheesh, K.; Jayavel, R., Synthesis and electrochemical properties of reduced graphene oxide via chemical reduction using thiourea as a reducing agent. Mater. Lett. 2013, 113, 5-8.

42. Wang, X.; Wang, J.; Wang, D.; Dou, S.; Ma, Z.; Wu, J.; Tao, L.; Shen, A.; Ouyang, C.; Liu, Q.; Wang, S., One-pot synthesis of nitrogen and sulfur co-doped graphene as efficient metal-free electrocatalysts for the oxygen reduction reaction. Chem. Commun. 2014, 50 (37), 4839-42.

43. Si, Y.; Samulski, E. T., Synthesis of water soluble graphene. Nano. Lett. 2008, 8 (6), 1679-82.

44. $\quad$ Ferrari, A. C.; Meyer, J. C.; Scardaci, V.; Casiraghi, C.; Lazzeri, M.; Mauri, F.; Piscanec, S.; Jiang, D.; Novoselov, K. S.; Roth, S.; Geim, A. K., Raman spectrum of graphene and graphene layers. Phys. Rev. Lett. 2006, 97 (18).

45. Tuinstra, F.; Koenig, J. L., Raman Spectrum of Graphite. J Chem. Phys. 1970, 53 (3), 1126-1130.

46. Lucchese, M. M.; Stavale, F.; Ferreira, E. H. M.; Vilani, C.; Moutinho, M. V. O.; Capaz, R. B.; Achete, C. A.; Jorio, A., Quantifying ion-induced defects and Raman relaxation length in graphene. Carbon 2010, 48 (5), 1592-1597.

47. Chen, S.; Zhu, J.; Wu, X.; Han, Q.; Wang, X., Graphene oxide--MnO2 nanocomposites for supercapacitors. ACS Nano 2010, 4 (5), 2822-30.

48. Santhosh, C.; Nivetha, R.; Kollu, P.; Srivastava, V.; Sillanpää, M.; Grace, A. N.; Bhatnagar, A., Removal of cationic and anionic heavy metals from water by 1D and 2D-carbon structures decorated with magnetic nanoparticles. Sci. Rep. 2017, 7 (1), 14107

49. Guo, X.; Du, B.; Wei, Q.; Yang, J.; Hu, L.; Yan, L.; Xu, W., Synthesis of amino functionalized magnetic graphenes composite material and its application to remove $\mathrm{Cr}(\mathrm{VI}), \mathrm{Pb}(\mathrm{II})$, $\mathrm{Hg}(\mathrm{II}), \mathrm{Cd}(\mathrm{II})$ and $\mathrm{Ni}(\mathrm{II})$ from contaminated water. J. Hazard. Mater. 2014, 278, 211-220.

50. Gu, W. T.; Sevilla, M.; Magasinski, A.; Fuertes, A. B.; Yushin, G., Sulfur-containing activated carbons with greatly reduced content of bottle neck pores for double-layer capacitors: a case study for pseudocapacitance detection. Energy Environ. Sci. 2013, 6 (8), 2465-2476.

51. Singhal, R.; Kalra, V., Using common salt to impart pseudocapacitive functionalities to carbon nanofibers. J. Mater. Chem. A 2015, 3 (1), 377-385.

52. Liu, Z.; Wu, Z.-S.; Yang, S.; Dong, R.; Feng, X.; Müllen, K., Ultraflexible In-Plane Micro-Supercapacitors by Direct Printing of Solution-Processable Electrochemically Exfoliated Graphene. Adv. Mater. 2016, 28 (11), 2217-2222. 